\title{
Designing Fuzzy Inference System to Diagnosis down Syndrome by Face Processing
}

\author{
Mohammad Mojtaba \\ Keikhayfarzaneh \\ Department of Psychology, \\ Young Researcher Club, \\ Zahedan Branch ,Islamic \\ Azad University, Zahedan, \\ Iran
}

\author{
B.Somayeh Mousavi \\ Department of Computer, \\ Young Researcher Club, \\ Zahedan Branch ,Islamic \\ Azad University, Zahedan, \\ Iran
}

\author{
Javad Khalatbari \\ Islamic Azad University, \\ Tonekabon Branch, \\ Department of Psychology \\ ,Tonekabon, Iran
}

\begin{abstract}
Various kind of mental illness is common in all parts of the globe, and all age groups. New diagnostic methods will be vital stage in reducing or eliminating these types of disease. In this paper novel fuzzy rule-base system is designed, and programmed by computer software, for diagnosis of Down Syndrom faces in an image. System input is an arbitrary color image. In first step face regions should be selected. An accurate face detection system is utilized which applies skin color, lip position, face shape information and ear texture properties, as the key parameters. After this stage, detected face regions are processing carefully by proposed fuzzy system, and some features such as face area and eye distance are investigating carefully. Finally the probability of being Down Syndrom is revealed by designed system. $98.33 \%$ correct detection is obtained applying this algorithm on various image databases. This system could be considered as the first step to device automatic system for diagnosis of illness and would help psychiatry.
\end{abstract}

\section{Keywords}

Mental illness, Down Syndrom, Face Detection, Fuzzy rulebased system.

\section{INTRODUCTION}

Mental illness are real, debilitating, and common, the burden of mental illness falls on individuals from all walks of life, all parts of the globe, and all age groups (WHO, 2001). Children and adolescents are not exempt. With half of all mentally ill adults exhibiting symptoms of mental illness by the age of 14 and three quarters by their midtwenties [1]. Table 1 shows the superfluity of mental disease among some nations [2].

Greater understanding of the developmental origins of mental illness offers hop that new diagnostic methods and better treatments will substantially reduce or eliminate mental illness [3], such as anxiety, attention deficit hyperactivity disorder (ADHD), autism, bipolar illness, depression, and schizophrenia. Beside common psychological methods, visual representation of mental illness, have a long history in psychiatry. The tradition of visually representing the mentally ill begins with Philippe Pinel's Medico-Philosophical Treatise on Mental Alienation, or Manca, published in 1801 [4]. Although it was commonplace by that time the pathology of insanity was reflected in the afflicted person's physical appearance, it was Pinel, famous for developing humane treatments for the insane, who was the first to provide illustrations to document his medical cases. His head illustrations are in the mode of earlier craniological studies, white the faces reflect the new studies of human physiognomy that had begun to appear in the eighteenth century. For Pinel, who sought to describe mental disease so as to classify them, head size and physiognomic properties were empirical indicators of an individual's mental state. Drawings of the insane, and the scale of normative appearance they established were an aide to diagnosis.

Pinel's student and co-worker, Jean Etienne Dominique Esquirol, expanded this method of visualizing and sorting the mentally ill. In his Dictionary of Medical Sciences (1812-22) and then more expansively in his textbook of 1838, Mental Maladies, which includes 27 engraved portrait drawings, Esquirol created an atlas of the posture and expression of the insane. He emphasized thet physiognomy was unsurpassed as a diagnostic tool and promised thet one day he "would provide diagnostic illustrations for every one of his reformulated nosological (disease classification categories)" . He did not make good on that promise, but others took up the project, most influentially the Scottish physician Alexander Morison. The case descriptions in Morison's 1840 atlas "The Physiognomy of Mental Disease" are accompanied by 108 lithographs, including contrasting pictures of the same patient "before" and "after" recovery. These illustrations purported to show the distinguishing physiognomic features for each of his diagnostic types. For Morison and others like him, to quote a commentator, "there was a readily identifiable 'face' for every type of madness" [5].

Existence of relationship between face processing and mental disease, was a motivation for us to device an automatic algorithm to diagnose the illness by face detection and processing systems. Face detection defined as detection and localization of human faces in images, has been challenging topic in human computer interaction (HCI) researches. Human face detection application is observable mainly in video surveillance, face recognition and image databases management [6].

In this paper both psychologcal and soft computing sciences are applied together to design, novel, robust, automatic system to diagnosis mental illness, based on the face features. To this aim, 
in first step, an accurate system is applied to detect face regions in an input image [7]. After this, a fuzzy inference system (FIS) is designed according to special face features of patient's face. Here we try our system to diagnosis Down Syndrome. Designed algorithm is the prime stage to develop an intelligent system, which could be so useful for assisting psychiatry, to diagnosis variety kind of mental illness by computer base processing.

The reminder of the paper is organized as follows. Section two includes: face detection and determining the probability of being ill systems, both of them performed by FIS. Finally some implementation results are presented and some ways to develop proposed system in future are suggested.

Table 1. Superfluity of Mental Disease

\section{PROPOSED METHOD}

The suggested method is a computer program which gets a sample face image as an input and determines the probability of being Down Syndrom patient. To achieve this goal, the exact face region should be localized. Then, considering some face features which are special in patient's faces, the fuzzy decision making system is designed, announce the probability of being Down Syndrom case. Whole described stages are converted to computer program using MATLAB software.

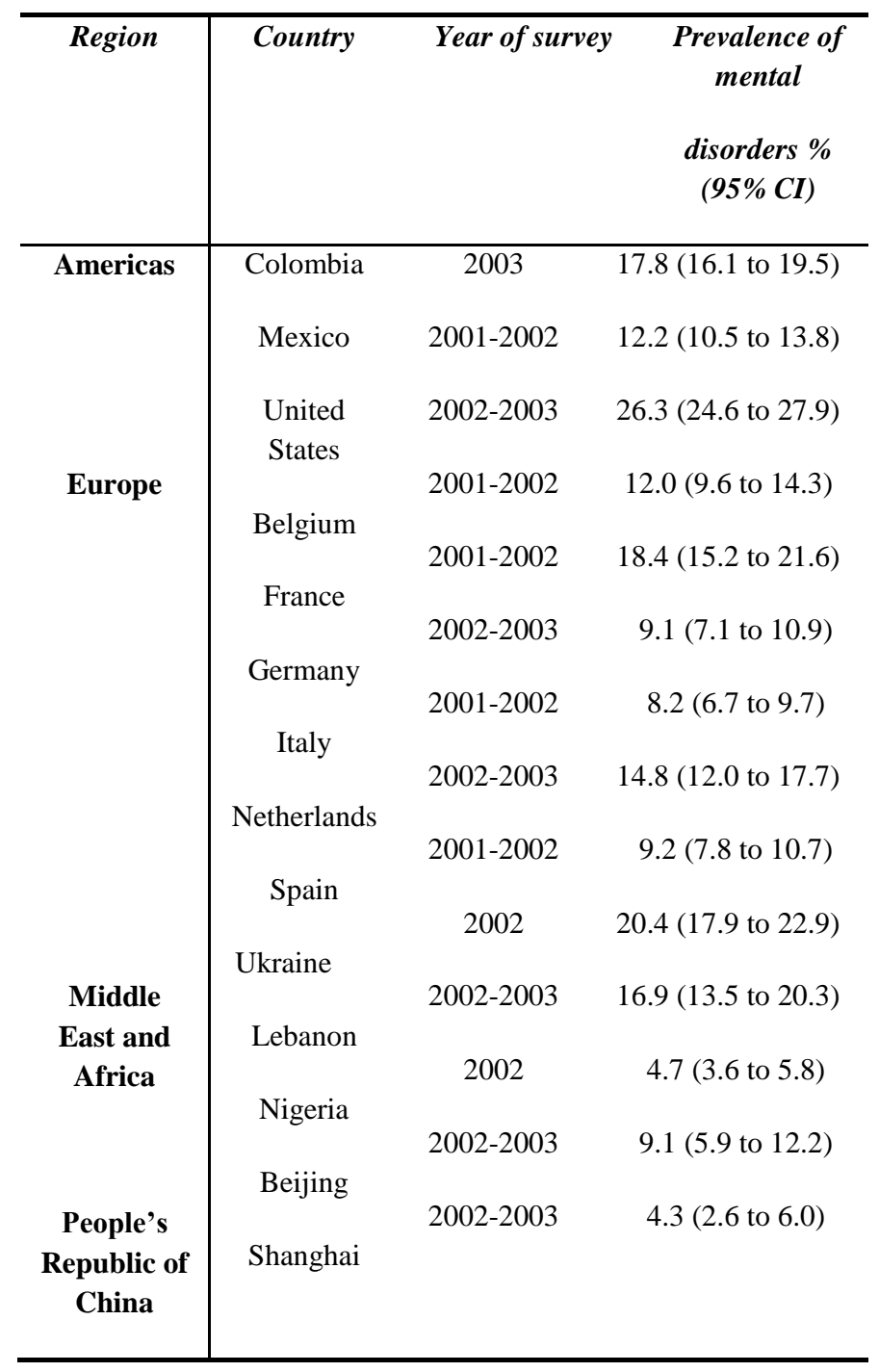

\subsection{FACE DETECTION SYSTEM}

In first stage to locate face area in an input image a robust pose independent algorithm is applied which has a priority of estimating face poses beside locate them in color images [7]. To design more efficient system, after detecting face area, it will be classifies as frontal, near frontal or profile. Pose estimation is very important in many face processing works including face modeling and face recognition. In general, face pose may present human physical states such as sleeping and concentration. As a result, it is very important in many real-life applications, such as monitoring attentiveness of drivers or automating camera management [8].

The block diagram of system is depicted in Fig. 1. As it can be seen in the first step the skin regions are introduced in an input image. Through each of the separated skin regions the face features, which are include lip and ear texture, are searched to materialize a robust pose/expression/lighting condition independent algorithm. This system is a fuzzy rule base one, optimized by genetic algorithms (GA).

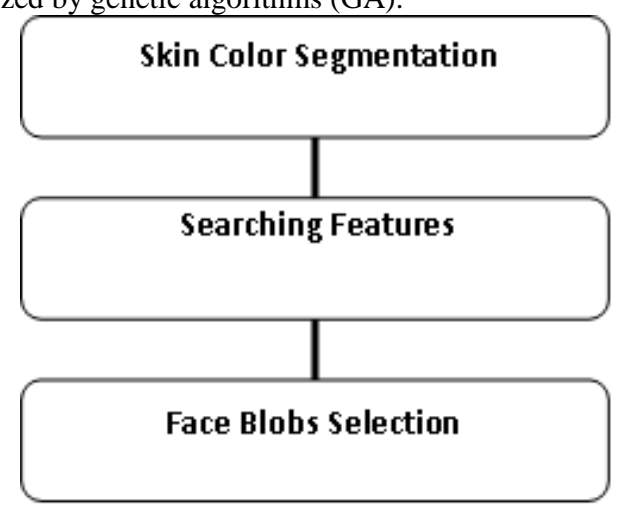

Fig. 1. Subsystems of proposed algorithm

Fuzzy theory provides a framework to materialize a fuzzy rule base system which contains the selection of fuzzy rules, membership functions, and the reasoning mechanism. Such a system has been applied to many disciplines such as control systems, decision making and pattern recognition [9], and in this paper it is supposed that such a system could overcome the complexity of the face detection problems, mainly are known as: variable conditions and diversity of human faces; as well as uncertainty of the basic face features.

However, it is often difficult for a human expert to define the fuzzy sets and fuzzy rules, used by these systems. GAs have proven to be a useful method to optimize membership functions of fuzzy sets used by these fuzzy systems [10].

\subsubsection{SKIN AND LIP COLOR SEGMENTATION}

Typically when skin color is utilized as a feature in face detection, three main questions would be arisen:

Firstly, what color space to choose. Secondly, how the skin color distribution should be modeled, and thirdly, what will be the way of processing of color segmentation results for face detection?

In the first step of the skin color segmentation, an appropriate color space should be selected. The transformation of RGB to perceptual color spaces is invariant to high intensity at white 
lights, ambient light and surface orientations relative to the light source. Consequently, they can be a so suitable choice for skin detection methods.

Using following non-linear equations, RGB color space transforms to HSI color space, where the intensity component is separated from the chrominance components:

$H=\cos ^{-1} \frac{\frac{1}{2}((R-G)+(R-B))}{\sqrt{\left((R-G)^{2}+(R-B)(G-B)\right)}}$

$S=1-3 \frac{\operatorname{Min}(R, G, B)}{R+G+B}$

$I=\frac{1}{3}(R+G+B)$

Although in many skin classification research the intensity component is omitted, 2D projection of $3 \mathrm{D}$ color distribution can not completely adapts itself to lighting variation. Furthermore, simply discarding luminance information affects the model's accuracy. To obtain optimum color space, different fractions of color space components are tried, and finally $\left(\frac{1}{5} H, S, \frac{2}{3} I\right)$ are chosen as the best.

After transforming the input image in to the selected color space, next step is finding the skin pixels. Mamdani fuzzy system used is a 1-input, 1-output system applying the Euclidean distance between the color of each pixel to the average skin color sub-space as an input, and the likelihood of being skin pixel as an output. Subtractive clustering [11] is applied on input space (contain 132000 skin and non skin pixels) to decide about the number of MFs and rules. Utilizing the obtained four clusters information and experimental knowledge, input and output MFs are designed. The semantic meaning, $\{$ Skin, Rather Skin, Low Probability Skin, Not Skin\}, assigned to clusters for better understanding.

To achieve the crisp output, centroid method is chosen, which is the most widely used one among all defuzzification approaches [9]. The membership functions (MFs) of the inputs and output is depicted in Fig. 2.

The result of applying such a system is the skin-likelihood image; that is, the gray-scale image whose gray values represent the likelihood of the pixel belonging to the skin. To make a binary image, the threshold value should be selected. Genetic Algorithm (GA) is used to find the optimized value. GAs are the most extended group of evolutionary techniques, which rely on the use of a selection, crossover and mutation operators [10]. The threshold value is applied as an input of the GA, whose fitness function compares the whole detected skin pixels in the sample image with the actual number of these pixels, and tries minimizing the different between them as much as possible.

An obtained value is 0.8964 which means that the pixel with $\% 89.64$ or more are regarded as the skin pixels. The binary image is formed by setting skin pixels to 1 and all other pixels to 0 . After that, morphological processing, consists of filling holes and opening followed by closing [12], is accomplished to acquire separate and connected regions. After labeling connected the components, each region is applied as an input for the next step, to find lip area in each skin region.

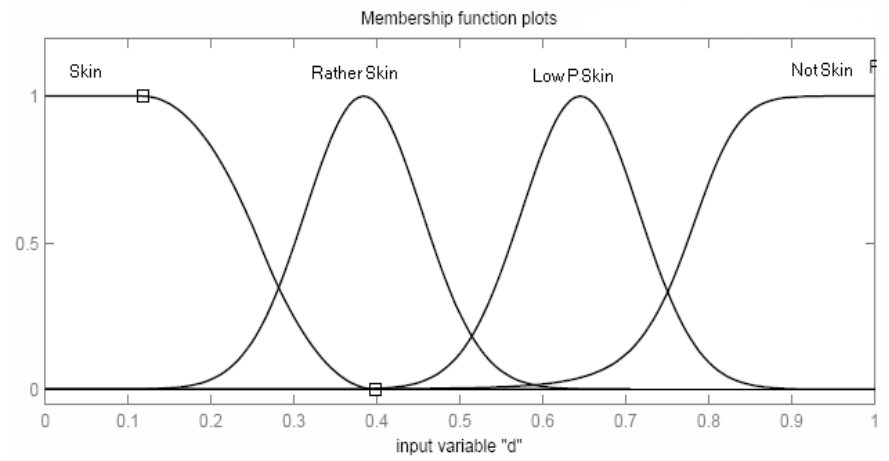

(a)

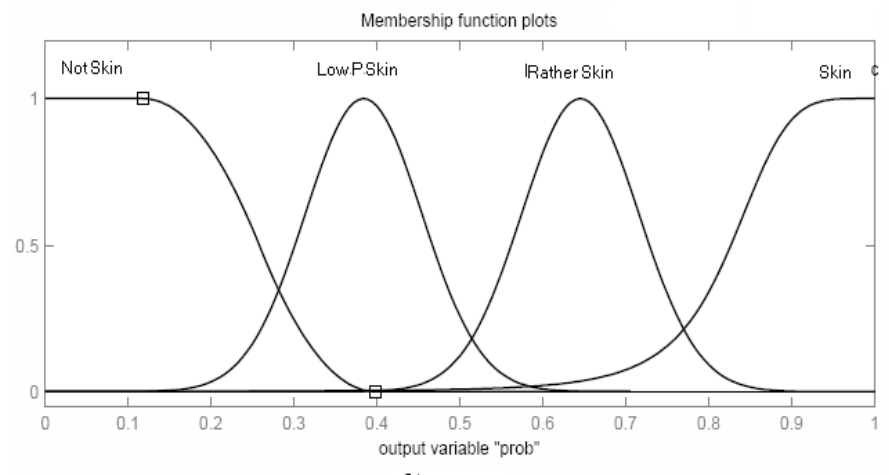

(b)

Fig. 2. (a) input and (b) output MFs for skin color segmentation fuzzy classifier

Lip color segmentation section, searches the whole skin region to find lip pixels. Finding lip pixels system describes as follows: The target region is transformed to normalized RGB color space. This color space is achieved using equation (11).

$$
\begin{aligned}
& r(x, y)=\frac{R(x, y)}{\operatorname{Max}(R, G, B)} \\
& g(x, y)=\frac{G(x, y)}{\operatorname{Max}(R, G, B)} \\
& b(x, y)=\frac{B(x, y)}{\operatorname{Max}(R, G, B)}
\end{aligned}
$$

This color space has been shown the best result in finding lip cases [13].

In normalised RGB color space and within the desired region, the $r$ - $g$ values are utilized as fuzzy system input. The properties and design process of this system are same as the skin color segmentation algorithm. The input space (contain 50700 lip and non lip pixels) is divided in three clusters utilizing subtractive clustering. 


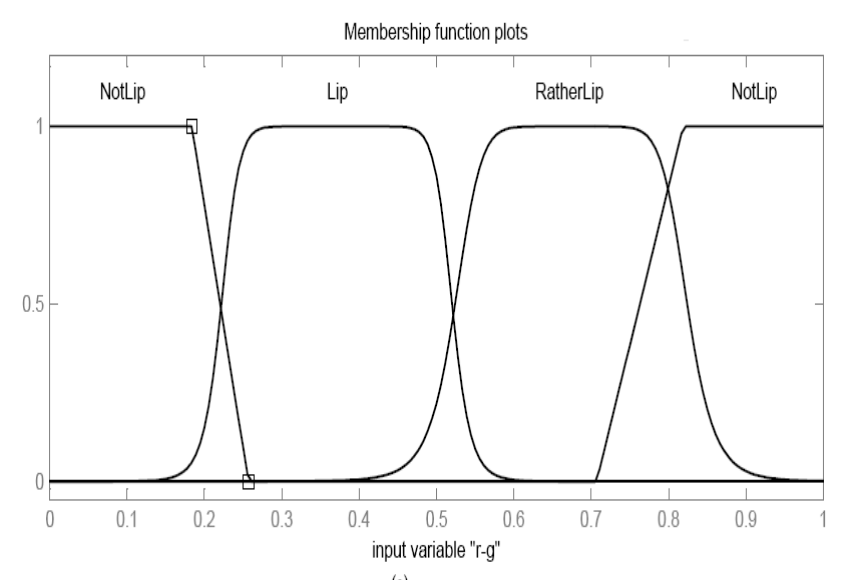

(a)

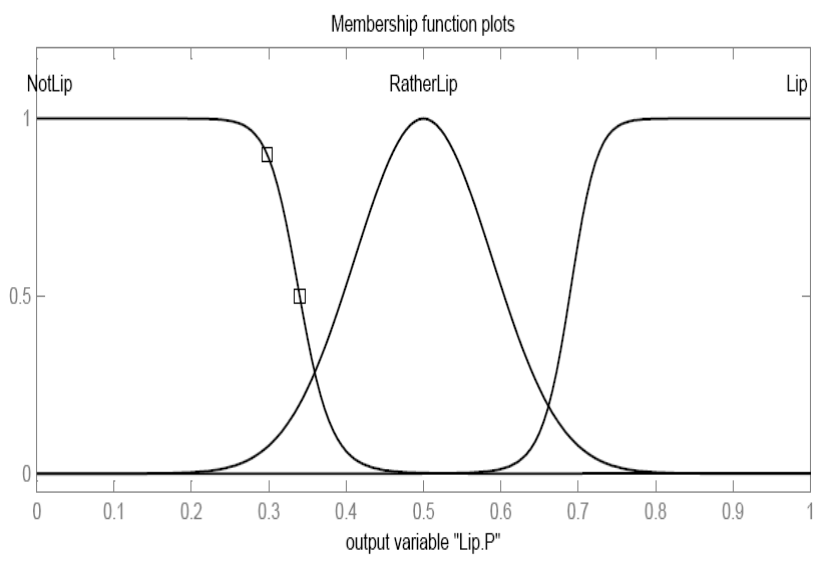

(b)

Fig. 3. (a) input and (b) output MFs for lip color segmentation fuzzy classifier

The input ( $\mathrm{r}-\mathrm{g})$ is recognized by linguistic terms: \{Lip, Rather Lip, Not Lip \}; likewise, for the output, linguistic terms are: \{Lip, Rather Lip, Not Lip\}. MFs are illustrated in Fig. 3.

Similar to skin detection system, the result is lip-likelihood image. $82.7 \%$ likelihood is chosen by GA, as threshold to make binary image of lip regions.

Filling the holes and erosion plus opening by reconstruction [12], are the morphological processing performed at the end of this stage. Fig. 4 shows the skin and lip detected areas in them.
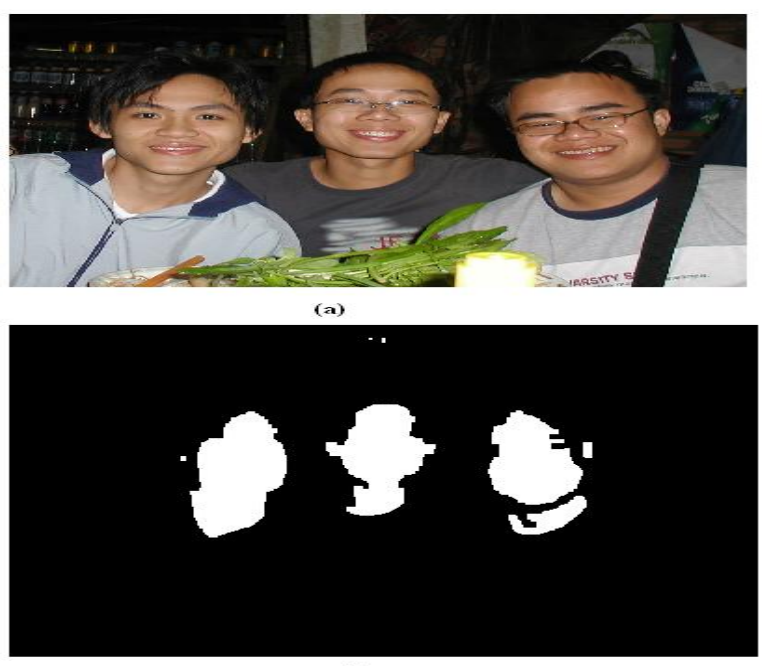

(b)

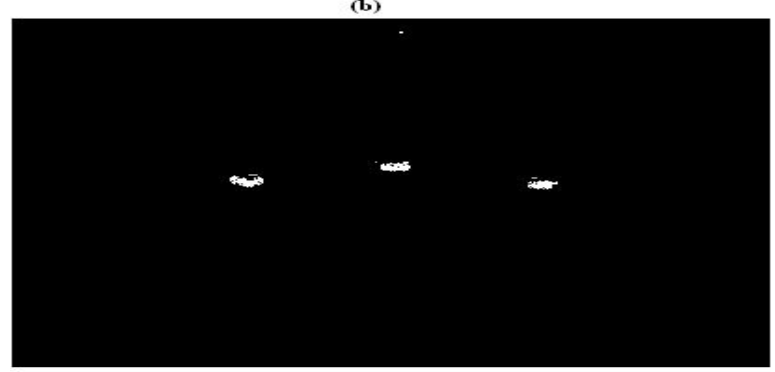

(c)

Fig. 4. (a) sample image, (b) skin color regions and (c) lip color regions.

\subsubsection{SELECTING FACE BLOBS}

In this algorithm, the frontal and near frontal face blobs would be selected in the skin regions. Decision making system for frontal and near frontal face detection is a fuzzy system with three inputs and one output. The first input (A) is the ratio of the lip area to the face area. The second input (B) is the tangent of angle between the maximum length line of face area and the minimum length line of the lip area. These two lines should be approximately parallel. Angles less than $30^{\circ}$ are acceptable. The third input (C) reveals the similarity of region shape to an ellipse. This ellipse estimates the face shape. Applying such inputs, the false positive of algorithm would be as minimum as possible. Utilizing 100 different face and non face regions, three subspaces introduced by subtractive clustering algorithm to make input space.

Output (Y) expresses likelihood of the region being face. The inputs and output MFs are illustrated in Fig. 5. The linguistic terms are: $\{$ Face, rather Face, Non Face $\}$ for both input and output MFs.

Regions with more than $74 \%$ likelihood are introduced as a frontal face. This value selects by GA, attempting to maximize the likelihood of being face for some faces blob samples. 

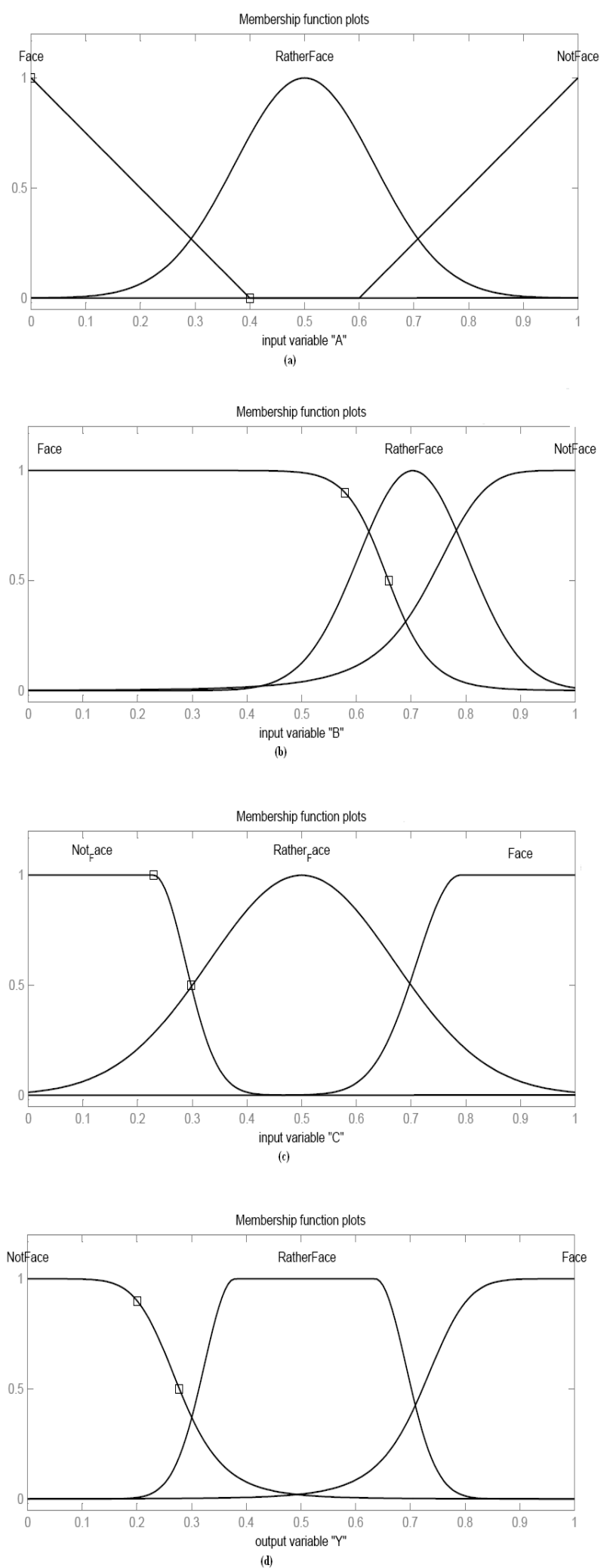

rig. s. (a), (v), (c) inputs and (a) output ivirs ior detecing frontal face candidate fuzzy system
This algorithm works successfully to select frontal and near frontal, in which the lip area could be detected, but it has low detection rate for profile faces [14]. So, to compensate this weakness, another algorithm, called profile face detecting algorithm, is designed and applied beside the frontal face detecting one to form robust system for various face pose.

To search profile faces among skin regions, the properties of ear texture is used. First of all, the ear location is estimated by its relative position to nose tip.

The suggested process of finding nose tip in [15], is altered to be size independent and used here. The new process is as follows: the $X$ values along each row which we first encounter a white pixel in the binary image is searched. With the median of these values $\left(X_{\text {median }}\right)$ the approximation $X$ value of the face contour is obtained. Then, the median of $Y$ values in the range of $X_{\text {median }}$ is calculated $\left(Y_{\text {median }}\right)$. In the last step, the $X$ value of nose tip is introduced as the minimum $X$ values in the range of:

$Y_{\text {median }}-(n / 2)<Y<Y_{\text {median }}+(n / 2)$

where $n$ is the number of $Y$ values in the range of $X_{\text {median }}$. This method avoids the possibility of locating hair or chin as the nose tip.

Since determining $X$ and $Y$ values of nose tip, the ear location is estimated by use of the distance between nose tip and the last white pixel, its $Y$ value equal to $Y_{\text {nose }}$. In the regions with high probability of ear existence, the properties of texture is checked.

After studying various texture classification methods, such as statistical properties of the intensity histogram (statistical moments) [16], Geometric Moment (GM) is chosen to check ear area.

Again, instead of crisp threshold, a fuzzy rule base system is designed based on the $\Phi_{1}$ and $\Phi_{2}$. These two values are employed as input and the probability of ear texture existence is produced as output. \{Ear, Not Ear\} are chosen as linguistic term for two detected cluster. Fig. 6 shows the MFs of such a system. If the existence of ear in the region of interest is verified $(67 \%$ likelihood is recognized as the optimized threshold), the blob is known as the profile face. 

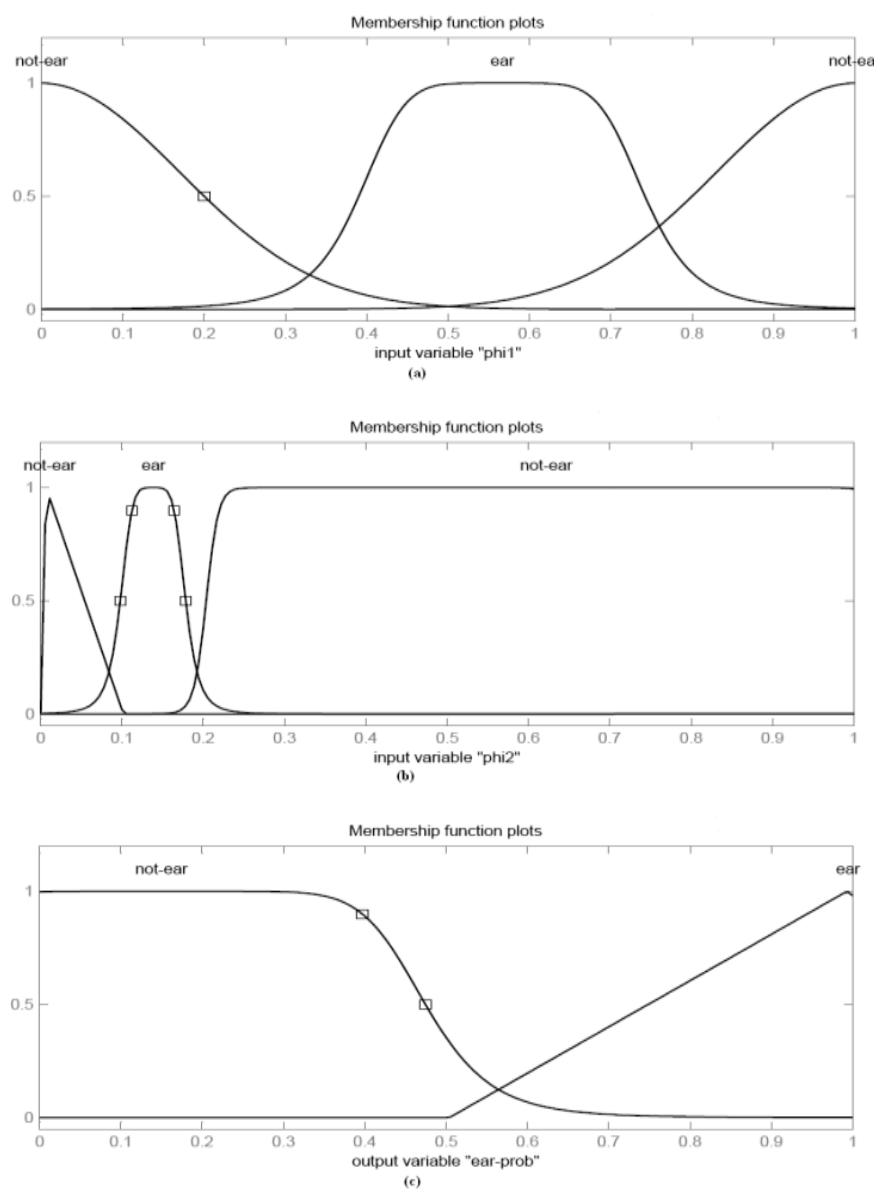

Fig. 6. (a) and (b) inputs and (c) output MFs for ear texture classification system

Finally whole obtained information, both profile and frontal face detecting systems are used to select faces in images. So, each skin blob entrances both these systems. Depending on each system response, there are 3 different states:

1. If none of frontal or profile systems identify the skin region as a face, it would be discarded.

2. If one of systems verifies the skin region as a face, that blob would introduce as the frontal or profile face.

3. If the outputs of both systems are positive for one skin region, it is a face but the challenging problem is its gesture.

The regions lie within third group, could be introduced as face ones, but to increase the accuracy of system, the pose detection approach is designed and applied for such cases. This approach works as follows:

The position of lip area in face region is estimated. The center of mass's $x$ values of face and lip areas are calculated and their distance is called $d_{l} . d_{2}$ is the length of face region minor axis. Regarding these two values, if $\mathrm{d}_{1}<\mathrm{d}_{2} / 4$ the detected face is frontal; otherwise, it is a profile one. Fig. 7 shows face detected regions by designed system.
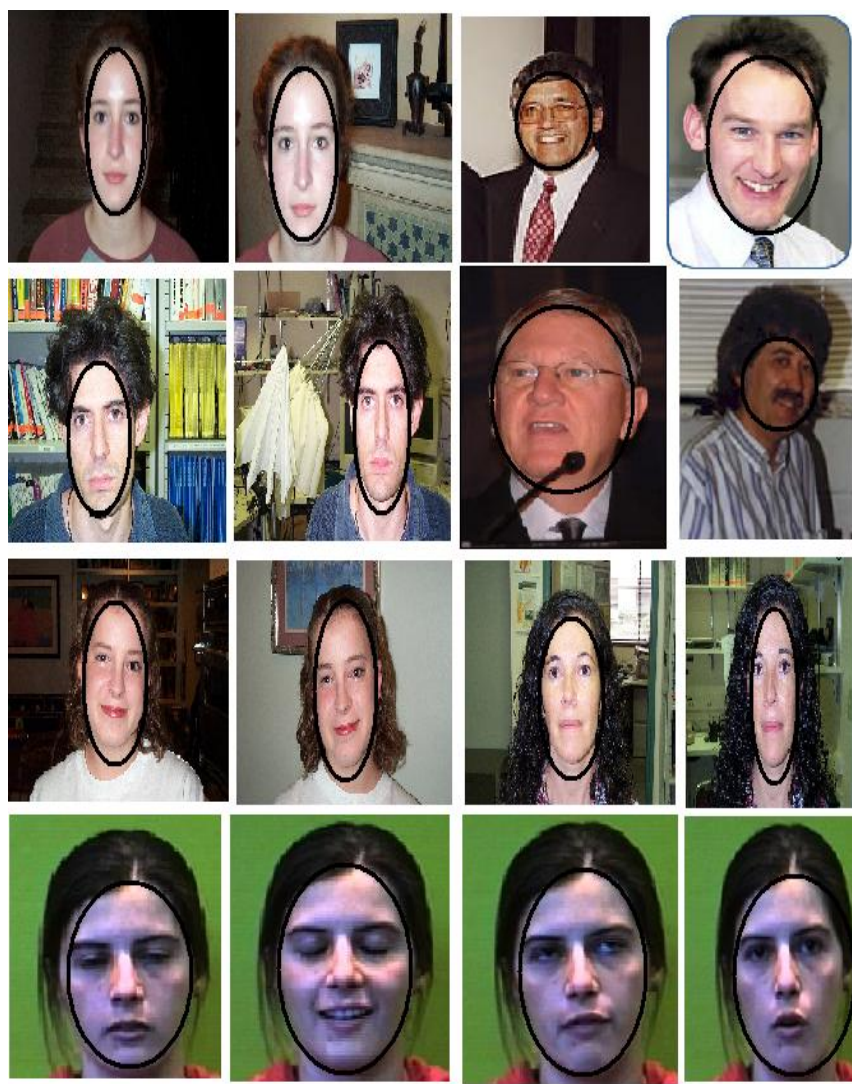

\subsection{DIAGNOSIS ILL FACES SYSTEM}

In this stage, another FIS is designed to determine the probability of being Down Syndrom patient. The designing process and system characteristics are as described in the previous section, just inputs and outputs would be altered. After investigating, comparing and face processing of various pictures, inputs are defined as follows:

A: The ratio of lip area to the face area. As the face area of patients is not normal, this value would be different for them.

B: Eye distance. Eyes' location is estimated relative to lip detected area, and distance between two eyes center is applied as the second input.

C: Number of black pixels in eyes region. This number is found more than usual for patient face picture.

Applying 100 different normal and Down Syndrom patient face pictures as an input space, three subspaces introduced by subtractive clustering algorithm.

Output (Y) expresses likelihood of the face region being patient. The inputs and output MFs are shown in Fig. 8. The linguistic terms are: $\{$ Normal, Rather Normal, Patient $\}$ for both input and output MFs. 

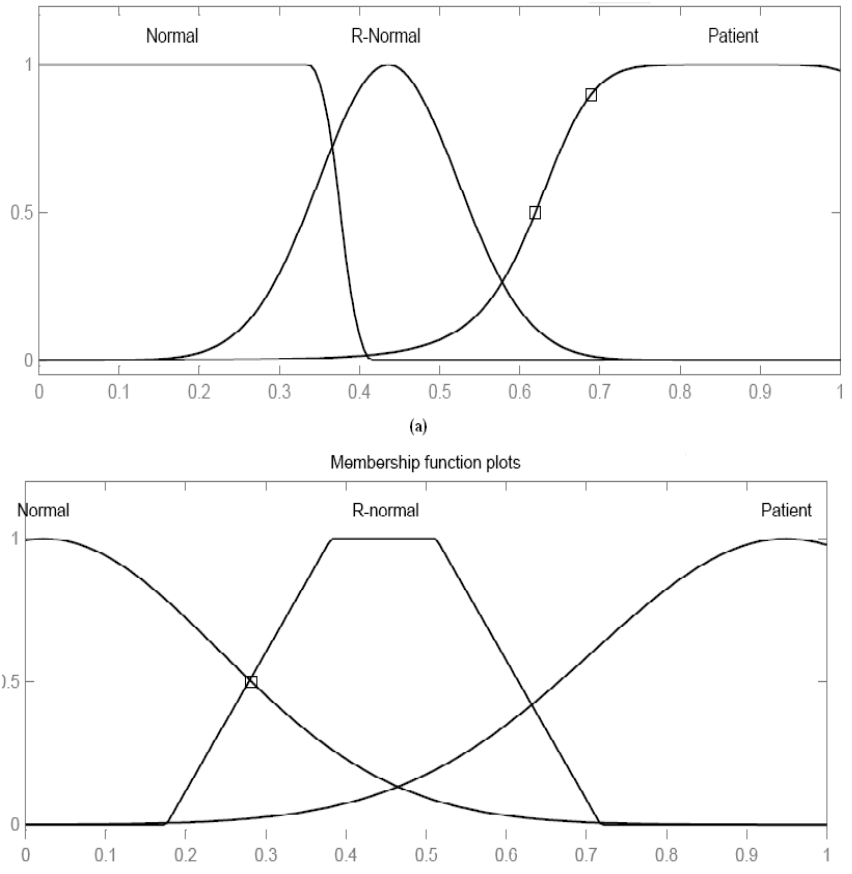

(b)

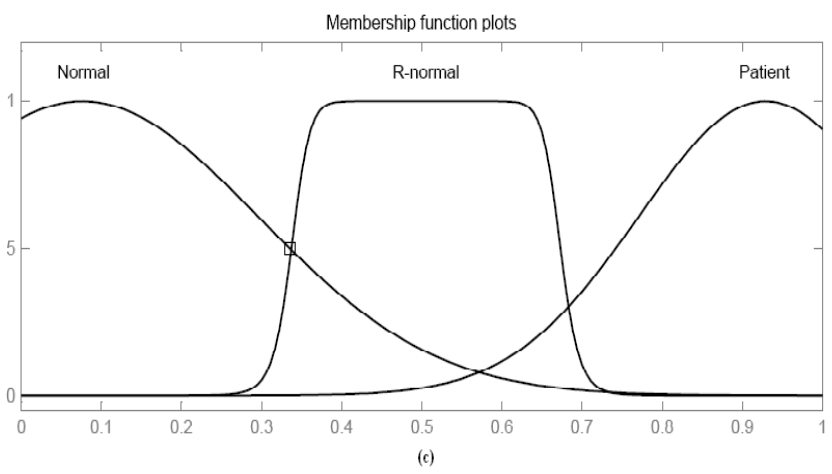

Momborship function plots

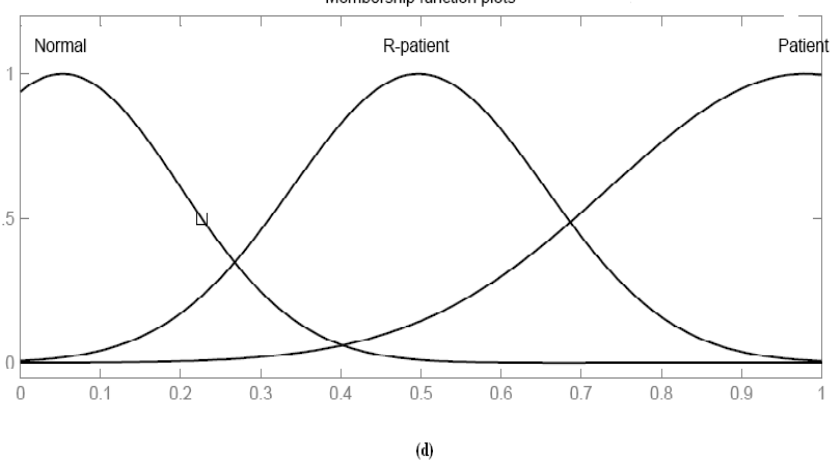

Fig. 8. (a), (b), (c) inputs and (d) output MFs for diagnosing patient candidate fuzzy system

\section{EXPERIMENTAL RESULTS}

To demonstrate effectiveness of proposed approach, an image dataset is formed, containing 300 face images (250 normal face images and 50 Down Syndrom face images). Various pose/ lighting condition/ facial expression/size $(125 \times 93$ to $296 \times 448$ pixels), and images of people from various racial origins are included in that. These images are gathered among:

1. Bao image dataset includes 370 face images from various races, mostly from Asia, with wide range of size and pose [17].

2. Caltech frontal face dataset, collected by Markus Weber at California institute of technology. There are 450 face images from 27 or so unique people under with different lighting/ expression/ backgrounds [18].

3. The IMM face database comprises 240 still images of 40 different human faces. The gender distribution is 7 females and 33 males and various facial expression and poses are contained [19].

4. As we need some Down Syndrom face image, and there were not any in face image database, these samples collected among various images in internet.

Firstly obtained detection rate for different databases is reported according to their pose (frontal/ near frontal/ profile) to prove the effectiveness of our face detection system. Table 2 shows these results.

After choosing face blobs, they would enter the second system. In this stage, the system's output shows the probability of being Down Syndrom cases. Successfully obtained results were achieved. For most of the patient cases, system output was more than $75 \%$. Just for two of them, considering $75 \%$ as threshold, the system failed to recognize. About normal faces, three cases introduced as patient wrongly. On balance, we could report $98.33 \%$ performance of our system which is really acceptable result. In Fig. 9 some examples of system outputs are shown.

\section{CONCLUSION}

Suggested method was a fuzzy inference system to diagnosis Down Syndrom by face processing. At first, faces regions were selected and processed by automatic computer program to determine if it is Down Syndrom face or not. Our system shows successfully $98.33 \%$ correct detection in a database contains 300 images. We are so hopeful and optimistic to device a perfect system for diagnosing any kind of illness by face processing that would be so helpful way to develop and improve psychiatry. 
Table 2. Experimental Results on Different Databases (DR: Detection Rate)

\begin{tabular}{|c|c|c|c|c|}
\hline$\overbrace{\text { Dataset }}^{\text {Head Pose }}$ & & Frontal & Near-Frontal & Profile \\
\hline \multirow{2}{*}{ Caltech } & Image's Number & 80 & - & - \\
\hline & $\mathrm{DR}(\%)$ & 98.75 & - & - \\
\hline \multirow{2}{*}{ Bao } & Image's Number & 40 & 20 & 10 \\
\hline & $\mathrm{DR}(\%)$ & 95 & 95 & 80 \\
\hline \multirow{2}{*}{$I M M$} & Image's Number & 60 & 40 & - \\
\hline & $\mathrm{DR}(\%)$ & 100 & 97.5 & - \\
\hline \multirow{2}{*}{ Added Images } & Image's Number & 40 & 10 & - \\
\hline & $\mathrm{DR}(\%)$ & 95 & 80 & - \\
\hline
\end{tabular}

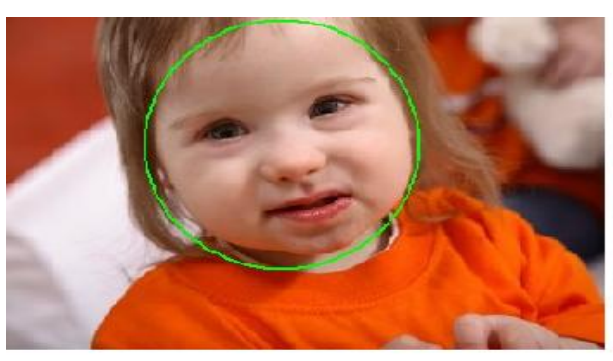

(a)

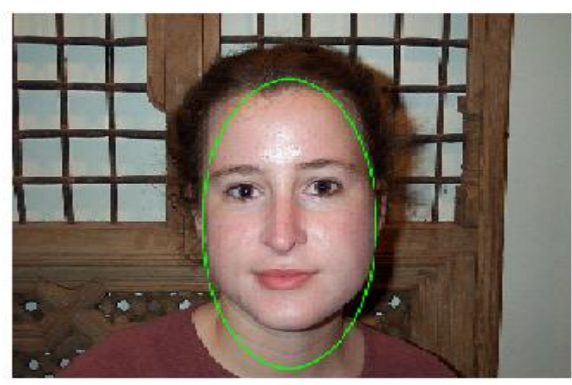

(d)

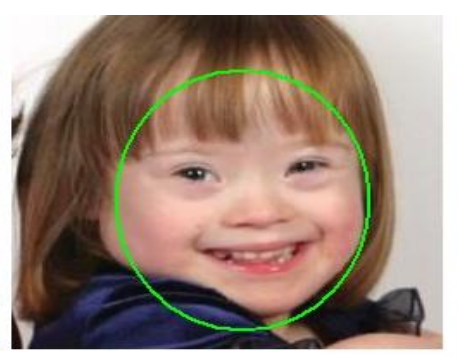

(b)

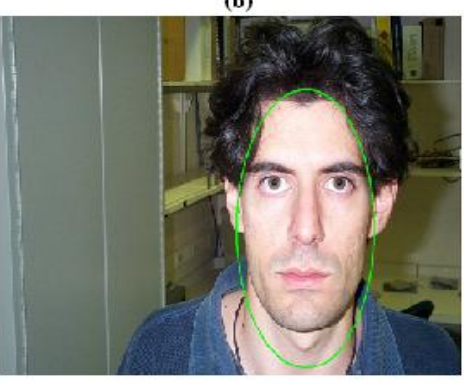

(e)

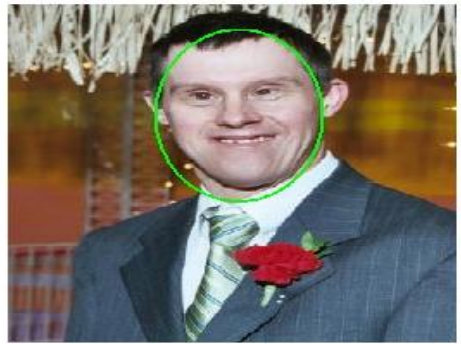

(c)

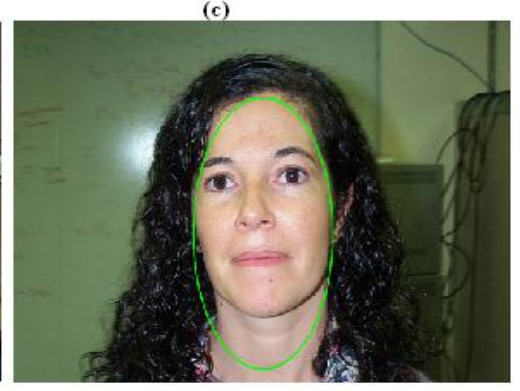

(f)

Fig. 9. Output system is respectively: $83.3 \%, 85 \%, 76.5 \%, 20 \%, 18 \%$ and $32.4 \%$ for (a-f) image samples

\section{REFERENCES}

[1] Kessler, R.C., Berglund, P., Demler, O., Jin, R., Merikangas, K.R., Walters, E.E. Lifetime prevalence and age-of-onset distributions of DSM-IV disorders in the National Comorbidity Survey Replication. Archives of General Psychiatry, 62(6): 593-602, 2005.

[2] Demyttenaere K, Bruffaerts R, Posada-Villa J, Worldwide prevalence of mental disorders is high but treatment needs are not always met, Evidence-Based Healthcare \& Public Health, 2004, pp. 359-361.
[3] NIMH available http://www.nimh.nih.gov/index.shtml

[4] Sander L. Gilman, Seeing the Insane (New York: John Wiley \& Sons, 1982).

[5] Janet Browne, "Darwin and the face of madness," The Anatomy of Madness: Essays in the History of Psychiatry, vol. 1, ed. W.F. Bynum, Roy Porter, and Michael Shepherd (New York: Tavistock, 1985)

[6] M.Yang, D.J.Kriegman, N.Ahuja, Detecting Face in Image: A Survey, IEEE Transaction on Pattern Analysis and Machine Intelligence, Vol.24, No.1, 2002. 
[7] P. Moallem, B.S. Mousavi, S.A. Monadjemi, A Novel Rule Base System for Pose independent Faces Detection, Applied Soft Computing, 2011, pp. 18011810.

[8] Q. Liu, Y. Ruj, A. Gupta and JJ Cadiz, Automating Camera Management for Lecture Room Environment, In: ACM CHI, 2000.

[9] S.N.Sivanandum, S.Sumathi, S.N.Deepa, 'Introduction to Fuzzy Logic Using MATLAB', (springer-verlag Berlin Heidelberg, 2007)

[10] S.N.Sivanandam S.N.Deepa, 'Introduction to Genetic Algorithms', (Springer-Verlag Berlin Heidelberg, 2008), pp. 165-209

[11] Agus Priyono, Muhammad Ridwan, Generation of Fuzzy Rules With Subtractive Clustering, Jurnal Teknologi, 2005, pp. 143-153.

[12] R.Davies, 'Machine Vision', (Morgan Kaufman, 2004)

[13] J.A.Dargham, A.Chekima, 'Lips Detection in the Normalised RGB Color Scheme', Information and
Communication Technologies, 2006, (1), pp.15461551.

[14] S. Mousavi, P. Moallem, M. Kavosh, Detecting Faces in Colour Images Using Fuzzy Rule Based Classifier, $2^{\text {nd }}$ Joint Congress on Fuzzy and Intelligent Systems, Tehran, Iran, 2008.

[15] P.Yan, K.W.Boyer, 'Biometric Recognition Using 3D Ear Shape', IEEE Transaction on Pattern Analysis and Machine Intelligence, 2007, 29, (8)

[16] S. Mousavi, P. Moallem, M. Kavosh, A Novel Fuzzy Rule Based Algorithm to Detect Faces with Various Pose, $17^{\text {th }}$ Iranian conference on Electrical engineering, Tehran, Iran, 2009.

[17] Face detection available at: http://www.facedetection.com.

[18] computational foundations of vision available at: http://www. vision.caltech.edu.

[19] The AAM Explorer available at: http://www.imm.dtu.dk/ aam/aamexplorer 